\title{
A novel algorithm for diagnosis of invasive pulmonary aspergillosis based on pentraxin 3 gene polymorphisms and its adjusted value among autoimmune diseases patients
}

\author{
Yuetian Yu ${ }^{1 \#}$, Chunyan Liu" ${ }^{2 \#}$, Cheng Zhu ${ }^{3 \#}$, Han Zhong ${ }^{4}$, Zhichun Gu${ }^{4}$, Chunhui Xu ${ }^{5}$, Chun Pan ${ }^{6}$, \\ Zhijun $\mathrm{Xu}^{7}$, Zhixiong $\mathrm{Wu}^{8}$, Wenjuan $\mathrm{Wu}^{9}$, Liangjing $\mathrm{Lu}^{10}$
}

${ }^{1}$ Department of Critical Care Medicine, Ren Ji Hospital, Shanghai Jiao Tong University School of Medicine, Shanghai, China; ${ }^{2}$ Department of Emergency, Minhang District Central Hospital, Shanghai, China; ${ }^{3}$ Department of Disease Prevention and Control, Ruijin Hospital, Shanghai Jiao Tong University School of Medicine, Shanghai, China; ${ }^{4}$ Department of Pharmacy, Ren Ji Hospital, Shanghai Jiao Tong University School of Medicine, Shanghai, China; ${ }^{5}$ Department of Laboratory Medicine, Institute of Hematology and Blood Diseases Hospital, Chinese Academy of Medical Science and Peking Union Medical College, Tianjin, China; ${ }^{6}$ Department of Critical Care Medicine, Zhongda Hospital, School of Medicine, Southeast University, Nanjing, China; ${ }^{7}$ Department of Critical Care Medicine, The Second Affiliated Hospital, Zhejiang University School of Medicine, Hangzhou, China; ${ }^{8}$ Department of Critical Care Medicine, Huadong Hospital, Fudan University, Shanghai, China; ${ }^{9}$ Department of Laboratory Medicine, Shanghai East Hospital, Tongji University School of Medicine, Shanghai, China; ${ }^{10}$ Department of Rheumatology, Ren Ji Hospital, Shanghai Jiao Tong University School of Medicine, Shanghai, China

Contributions: (I) Conception and design: Y Yu, C Zhu; (II) Administrative support: L Lu, W Wu; (III) Provision of study materials or patients: C Pan, Y Yu, Z Wu; (IV) Collection and assembly of data: C Zhu, Y Yu, H Zhong, Z Xu; (V) Data analysis and interpretation: H Zhong, Y Yu, Z Gu; (VI) Manuscript writing: All authors; (VII) Final approval of manuscript: All authors.

\#These authors contributed equally to this work.

Correspondence to: Liangjing Lu. Department of Rheumatology, Ren Ji Hospital, Shanghai Jiao Tong University School of Medicine, 145 Middle Shandong Road, Shanghai 200001, China. Email: lu_liangjing@163.com; Wenjuan Wu. Department of Laboratory Medicine, Shanghai East Hospital, Tongji University School of Medicine, 1800 Yuntai Road, Shanghai 200123, China. Email: wwj1210@126.com; Zhixiong Wu. Department of Critical Care Medicine, Huadong Hospital, Fudan University, 221 West Yan'an Road, Shanghai 200040, China. Email: Zhixiong.woo@gmail.com.

Background: Invasive pulmonary aspergillosis (IPA) is a rapidly progressive and fatal disease for those with autoimmune diseases. The performance of existing diagnostic tools is unsatisfactory, and a novel algorithm based on pentraxin 3 (PTX3) gene polymorphisms with adjusted PTX3 and galactomannan (GM) values is urgently needed.

Methods: Levels of PTX3 and GM were measured in the bronchoalveolar lavage fluid (BALF) and blood samples of patients who had autoimmune diseases with IPA between June 2017 and June 2021. Urea dilution was applied internally to correct the real BALF PTX3 and GM values. Three singlenucleotide polymorphisms (SNPs; rs1840680, rs2305619, and rs3816527) in the PTX3 gene were detected by polymerase chain reaction direct sequencing, and their associations with IPA were evaluated. Receiver operating characteristic (ROC) curves based on different variables were generated to determine the best algorithm for IPA diagnosis.

Results: This study enrolled 50 patients with IPA and 100 without IPA in the control groups (comprising 50 patients with Aspergillus airway colonization and 50 patients with bacterial pneumonia). The levels of adjusted BALF PTX3 and GM were higher in the IPA group than in the control groups $(\mathrm{P}<0.05$, respectively). For diagnosing IPA, the best adjusted cutoff value for PTX in BALF was $14.5 \mathrm{ng} / \mathrm{mL}$ and the best adjusted cutoff value for GM in BALF was 2.5 optical density index (ODI). The SNP rs1840680 AA homozygote was associated with a higher risk of IPA [odds ratio (OR) 18.86, $95 \%$ confidence interval (CI): 7.96-44.69; $\mathrm{P}<0.01$ ], while no genotypic distribution differences were observed for the other 2 SNPs (rs2305619 and rs3816527). Six algorithms were established based on PTX3 gene polymorphisms. The algorithm consisting of PTX3 gene polymorphisms with adjusted BALF PTX3 and BALF GM values demonstrated the best diagnostic performance (sensitivity $90.03 \%$; specificity $97.09 \%$; area under the curve 0.94 ). 
Conclusions: It was revealed that our new algorithm based on PTX3 gene polymorphisms combined with adjusted BALF GM and BALF PTX3 values performed well in diagnosing IPA.

Keywords: Invasive pulmonary aspergillosis (IPA); pentraxin 3 (PTX3); gene polymorphisms; galactomannan (GM); autoimmune diseases

Submitted Aug 03, 2021. Accepted for publication Dec 24, 2021.

doi: 10.21037/atm-21-4017

View this article at: https://dx.doi.org/10.21037/atm-21-4017

\section{Introduction}

Invasive pulmonary aspergillosis (IPA) is a rapidly progressive disease which may lead to fatal illness and poor prognosis (1). With the rapidly increasing application of molecular detection methods in clinical practice, the disease has generated considerable attention over the last decade (2). Furthermore, with a better understanding of the pathogenesis of IPA, more nonneutropenic susceptible hosts of the disease have been identified, such as those with influenza, coronavirus disease 2019 (COVID-19), or autoimmune diseases (3).

The diagnostic algorithm for aspergillosis in the intensive care unit (AspICU) has been widely used in the ICU to distinguish airway Aspergillus colonization from IPA; however, no specific biomarker is included in this criterion, and Aspergillus must be isolated from the respiratory tract specimens at least once, which leads to missed diagnosis or misdiagnosis (4). Galactomannan (GM) in bronchoalveolar lavage fluid (BALF) is recommended by several practice guidelines as an accurate marker for the diagnosis of IPA, yet there is no consensus on what the best cutoff value is (1-3). Our previous study has indicated that urea might be helpful as an internal parameter to correct the BALF GM value, but the sensitivity is still unsatisfactory (5). Pentraxin 3 (PTX3) belongs to a superfamily of conserved proteins and is closely related to alveolar injury. Furthermore, recent reports have suggested that the single-nucleotide polymorphisms (SNPs) of the protein are associated with susceptibility to IPA; however, most studies only focus on patients with hematopoietic stem cell transplantation (HSCT) $(6,7)$.

Thus, a reliable algorithm with specific markers and SNPs is required to accurately diagnose IPA. We hypothesize that a new diagnostic model consisting of PTX3 gene polymorphisms, PTX3-adjusted concentrations in BALF, and features of chest computerized tomography (CT) may have significant diagnostic value for IPA. We present the following article in accordance with the STARD reporting checklist (available at https://dx.doi.org/10.21037/ atm-21-4017).

\section{Methods}

\section{Research briefs}

This multi-center, prospective, observational study was conducted in eastern China at 6 tertiary teaching hospitals from June 2017 to June 2021. The Ethics Committee of Shanghai Jiao Tong University approved the study (No. 2016-Clinical-Res-083), and written informed consent was obtained from all the inpatient participants or their next of kin. The study was conducted in accordance with the Declaration of Helsinki (as revised in 2013).

\section{Study population}

Hospitalized patients with autoimmune diseases who were diagnosed with IPA and aged between 18 and 80 years were included in the study. Patients were excluded if they (I) had received prophylactic anti-Aspergillus therapy after admission (II) were in a severe hypoxic state and could not withstand tracheoscopy, and (III) were dying and not expected to survive for more than 48 hours. Propensity score matching (PSM) was performed by gender and underlying disease (1:1:1). Thus, those with Aspergillus airway colonization or with bacterial pneumonia were enrolled into the control groups of our study.

\section{Disease definitions}

IPA was defined based on the current practice guidelines of the Infectious Diseases Society of America (IDSA) (1). Those patients with a positive result of Aspergillus culture from airway secretion samples and without any clinical symptom were defined as having Aspergillus airway colonization. Bacterial pneumonia was defined based on the guidelines 
for hospital-acquired and ventilator-associated pneumonia management (8). Autoimmune diseases were diagnosed according to the guidelines of the Society for Rheumatology (9-11).

\section{Sample collection and detection}

Fiberoptic bronchoscopy and bronchoalveolar lavage (BAL) were performed based on the practice guidelines of the British Thoracic Society (BTS; 2013). The segments with severe lesions revealed by chest CT were selected. A total amount of $100 \mathrm{~mL}\left(50 \mathrm{~mL}^{*}\right)$ of saline solution was instilled into the bronchia and aspirated gently (12). BALF samples were centrifuged at $400 \mathrm{~g}$ for $20 \mathrm{~min}$ and were stored in a sterile closed-collection tube for further testing (including urea, GM, fluorescence staining, microscopic examination, and culture). The levels of PTX3 and GM in the BAL and plasma/serum were measured by an enzymelinked immunosorbent assay (ELISA) according to the manufacturer's instructions.

\section{Genotyping strategy and DNA extraction}

Three SNPs (rs1840680, rs2305619, and rs3816527) were selected as the targets, based on previous publications and information from the GenBank (https://www.ncbi.nlm.nih. gov/snp/?term=PTX3) $(6,13,14)$. The selected SNPs were located within the coding region of the PTX3 gene and thus may possibly influence protein synthesis. Peripheral blood from each enrolled patient was collected, and genomic DNA were extracted from the peripheral blood using a genomic DNA extraction kit according to the manufacturer's recommendations. All genomic DNAs were stored in a freezer at $-80{ }^{\circ} \mathrm{C}$ until further analysis. Polymerase chain reaction (PCR) amplification was performed in an Eppendorf Mastercycler Pro S thermocycler (Eppendorf, Hamburg, Germany).

\section{Statistical analysis}

Categorical variables are presented as counts (n) and percentages (\%). They were compared using the chi-square $\left(\chi^{2}\right)$ test or the Fisher's exact test when the sample size was less than 5 . Normally distributed variables were compared using the Student's $t$-test and are expressed as means and standard deviation (SD), whereas nonnormally distributed data were compared using the Wilcoxon rank sum test and are reported as medians [with interquartile range (IQR)].
In the study, a two-sided $\mathrm{P}$ value $<0.05$ was considered statistically significant.

All the data from the electronic medical record system and the laboratory were analyzed with SPSS v. 21.0 (IBM Corporation, Armonk, NY, USA) and free R software v. 3.6.2 (https://www.r-projec.org). The receiver operating characteristic curve (ROC) was generated with MedCalc Statistical Software v. 15.2.2 (MedCalc Software bvba, Ostend, Belgium), and the area under the curve (AUC) was calculated to assess diagnostic efficacy. Other figures were drawn by GraphPad Prism software v. 9.1 (San Diego, California, USA).

\section{Results}

\section{Patient characteristics}

Of the 66 autoimmune diseases patients with IPA who were screened, 50 were enrolled in the study. Primary diseases of the patients included systemic lupus erythematosus (SLE) $(\mathrm{n}=24)$, antineutrophil cytoplasmic antibody (ANCA)associated vasculitis $(\mathrm{n}=15)$, and dermatomyositis $(\mathrm{n}=11)$. PSM was performed based on gender and underlying disease (1:1:1; Figure 1). A total of 50 patients with Aspergillus airway colonization and another 50 patients with bacterial pneumonia were included in the control groups. The demographics and clinical characteristics of all the patients are shown in Table 1. It was revealed that more patients with IPA underwent immunosuppressive therapy (64\%) and corticosteroid treatment $(90 \%)$ than the patients without IPA. The lymphocyte count [median $0.43 \times 10^{9} / \mathrm{L}$; IQR, $\left.(0.21-0.63) \times 10^{9} / \mathrm{L}\right]$ and the $\mathrm{T}$ helper $(\mathrm{Th}) \mathrm{CD}^{+}$cell count (median 164 cells $/ \mu \mathrm{L}$; IQR, $129-253$ cells $/ \mu \mathrm{L}$ ) in the IPA group were lower than those in the control groups $(\mathrm{P}<0.01$, respectively). The 30-day mortality in the IPA group was higher than that in the Aspergillus airway colonization group and the bacterial pneumonia group (36\% vs. $8 \%$. vs. $18 \%$; $\mathrm{P}<0.01$; Figure 2).

\section{BALF dilution and value of GM and PTX3 values}

The blood samples of 50 autoimmune diseases patients (SLE: $\mathrm{n}=24$; ANCA-associated vasculitis: $\mathrm{n}=15$; dermatomyositis: $\mathrm{n}=11$ ) without any infectious diseases and Aspergillus airway colonization were detected to analyze the baseline levels of serum GM and plasma PTX3. In these 50 healthy patients, it was revealed that the serum GM levels were all below 0.5 optical density index (ODI) and that the median plasma 


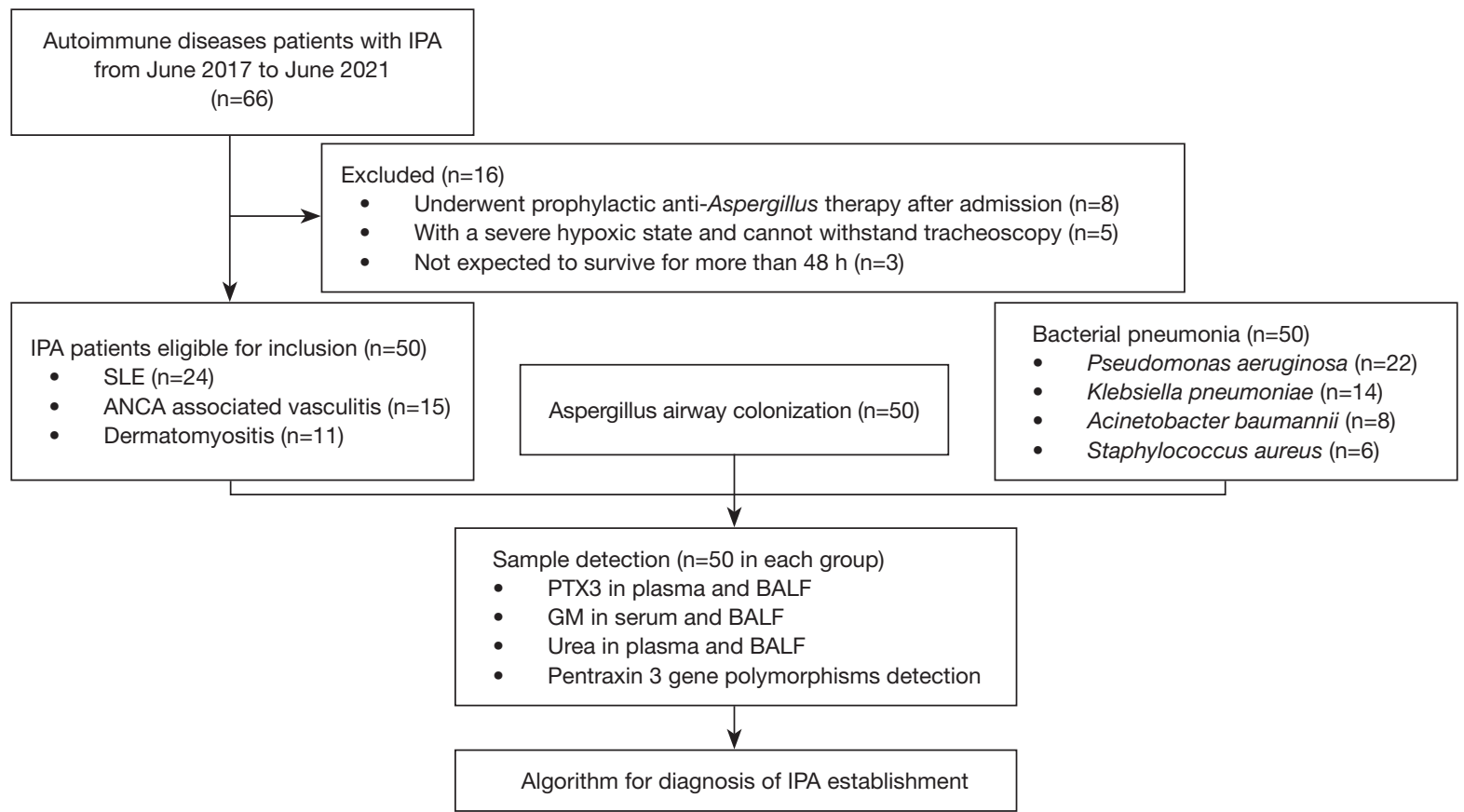

Figure 1 Flowchart of the study. ANCA, Anti-neutrophil cytoplasmic antibodies; BALF, bronchoalveolar lavage fluid; GM, galactomannan; IPA, invasive pulmonary aspergillosis; SLE, systemic lupus erythematosus; PTX3, pentraxin 3.

Table 1 Demographics and clinical characteristics of IPA and Non-IPA groups

\begin{tabular}{|c|c|c|c|c|}
\hline Characteristics & IPA $(n=50)$ & Aspergillosis colonization $(n=50)$ & Bacterial pneumonia $(n=50)$ & $P$ value \\
\hline Age, years ${ }^{a}$ & 37 [23-58] & $41[25-61]$ & 42 [25-59] & 0.28 \\
\hline Primary disease ${ }^{c}$ & & & & 1.00 \\
\hline SLE & $24[48]$ & $24[48]$ & $24[48]$ & \\
\hline ANCA-associated vasculitis & $15[30]$ & $15[30]$ & $15[30]$ & \\
\hline APACHE II score ${ }^{a}$ & 19 [13-26] & $12[8-17]$ & 16 [12-21] & 0.03 \\
\hline SOFA score ${ }^{a}$ & $8[5-11]$ & $4[2-7]$ & 7 [4-13] & 0.02 \\
\hline Immunosuppressive therapy $^{c}$ & 32 [64] & 17 [34] & $15[30]$ & $<0.01$ \\
\hline $\mathrm{PaO}_{2} / \mathrm{FiO}_{2}(\mathrm{mmHg})^{\mathrm{a}}$ & $178[101-257]$ & 224 [154-262] & 162 [113-235] & 0.03 \\
\hline High-flow nasal cannula ${ }^{c}$ & $15[30]$ & $7[14]$ & $18[36]$ & 0.04 \\
\hline Mechanical ventilation $^{c}$ & $24[48]$ & $6[12]$ & $12[24]$ & $<0.01$ \\
\hline 30-day mortality ${ }^{c}$ & $18[36]$ & $4[8]$ & 9 [18] & $<0.01$ \\
\hline
\end{tabular}

${ }^{a}$, medians [interquartile range]; ${ }^{b}$, means \pm standard deviations; ${ }^{c}, \mathrm{n}$ [\%]. IPA, invasive pulmonary aspergillosis; BMI, body mass index; APACHE II, acute physiology and chronic health evaluation II; SOFA, sequential organ failure assessment; SLE, systemic lupus erythematosus; ANCA, anti-neutrophil cytoplasmic antibodies. 


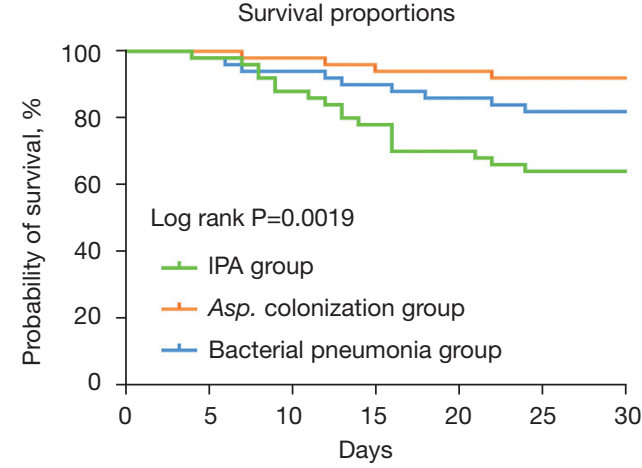

Figure 2 Thirty-day survival curves of the patients with or without IPA. IPA, invasive pulmonary aspergillosis.

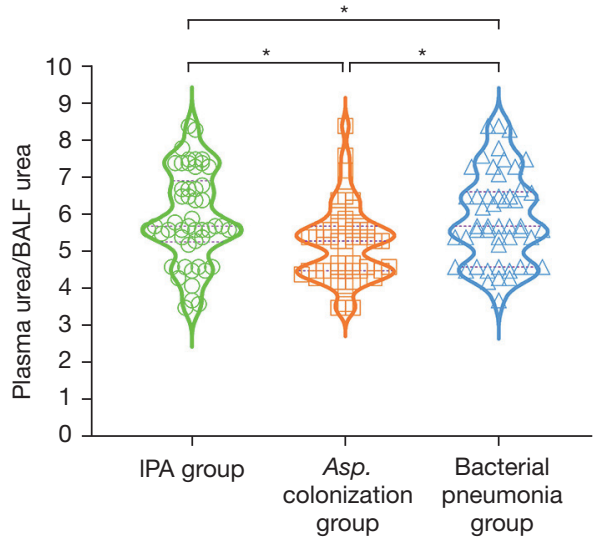

Figure 3 Comparison of dilutions of bronchoalveolar lavage fluid by urea among three different groups. *, $\mathrm{P}<0.05$. BALF, bronchoalveolar lavage fluid; IPA, invasive pulmonary aspergillosis.

PTX3 level was $0.23 \mathrm{ng} / \mathrm{mL}$ (IQR, $0.17-0.35 \mathrm{ng} / \mathrm{mL}$ ).

Urea analysis was performed as an internal parameter, and the plasma urea to BALF urea ratio was applied to assess the dilution of BALF. As was shown in Figure 3, the values of the plasma urea to BALF urea ratio in the IPA group (median 5.62; IQR, 5.37-6.71) and in the bacterial pneumonia group (median 5.71; IQR, 4.66-6.56) were higher than those in the aspergillosis airway colonization group (median 5.31; IQR, 4.51-5.73; $\mathrm{P}<0.01$ ). The levels of GM and PTX3 in blood and BALF were also detected, and the adjusted value of the biomarkers in BALF was calculated as follows:
It was revealed that in the IPA group the adjusted BALF PTX3 value was higher than the unadjusted BALF PTX3 value (median $23.69 \mathrm{ng} / \mathrm{mL}$; IQR, 18.56-26.62 ng/mL vs. median $11.02 \mathrm{ng} / \mathrm{mL}$; IQR, 7.43-13.23 ng/mL) and the adjusted BALF GM value was higher than the unadjusted BALF GM value (median 4.58 ODI; IQR, 3.22-5.64 vs. median 1.34 ODI; IQR, 0.96-1.76; $\mathrm{P}<0.01$; Figure 4).

\section{Correlation of PTX3 and GM between different samples}

Correlation analysis was performed, and the adjusted BALF PTX3 value was positively associated with the plasma PTX3 value $\left(\mathrm{Y}=18.60+0.78 \mathrm{X} ; \mathrm{R}^{2}=0.315 ; \mathrm{P}=0.009\right)$. In addition, it was also shown that the adjusted BALF GM value was positively associated with the serum $G M$ value $(\mathrm{Y}=2.918$ $\left.+1.419 \mathrm{X} ; \mathrm{R}^{2}=0.442 ; \mathrm{P}<0.001\right)$. No correlation was found between the biomarkers in the unadjusted BALF value and the blood value ( $>0.05$; Figure 5$)$.

\section{Diagnostic performance of biomarkers for IPA}

As one of the commonly used biomarkers to diagnose IPA, GM is commonly recommended, yet no standard cutoff value exists. Thus, the diagnostic performances of the different GM values were tested. It was revealed that when the BALF GM value was defined as $>0.8 \mathrm{ODI}$, better diagnostic accuracy was likely (sensitivity $80.02 \%$; specificity 78.21\%; AUC 0.79; Table 2 and Figure S1). The ROCs of different biomarkers are shown in Figure 6. The best cutoff value of adjusted BALF PTX3 was $14.51 \mathrm{ng} / \mathrm{mL}$, which yielded a sensitivity of $72.12 \%$ and a specificity of $97.82 \%$. A cutoff value of 2.51 ODI for the adjusted BALF GM resulted in an AUC of 0.85 (Table 3 and Table S1).

\section{Association between PTX3 genetic variants and the risk of aspergillosis}

The genotypic distributions of SNPs (rs1840680, rs2305619, and rs3816527) among the enrolled patients were detected. Genotypic differences in rs 1840680 between the IPA group and the other 2 groups were statistically significant $(\mathrm{P}<0.01)$; however, no such difference was found for the other 2 SNPs (rs2305619 and rs3816527). A regression model was performed for SNP rs1840680

$$
\text { Adjusted BALF GM or PTX3 value }=\text { BALF GM or PTX3 value } * \frac{\text { Plasma urea value }}{\text { BALF urea value }}
$$



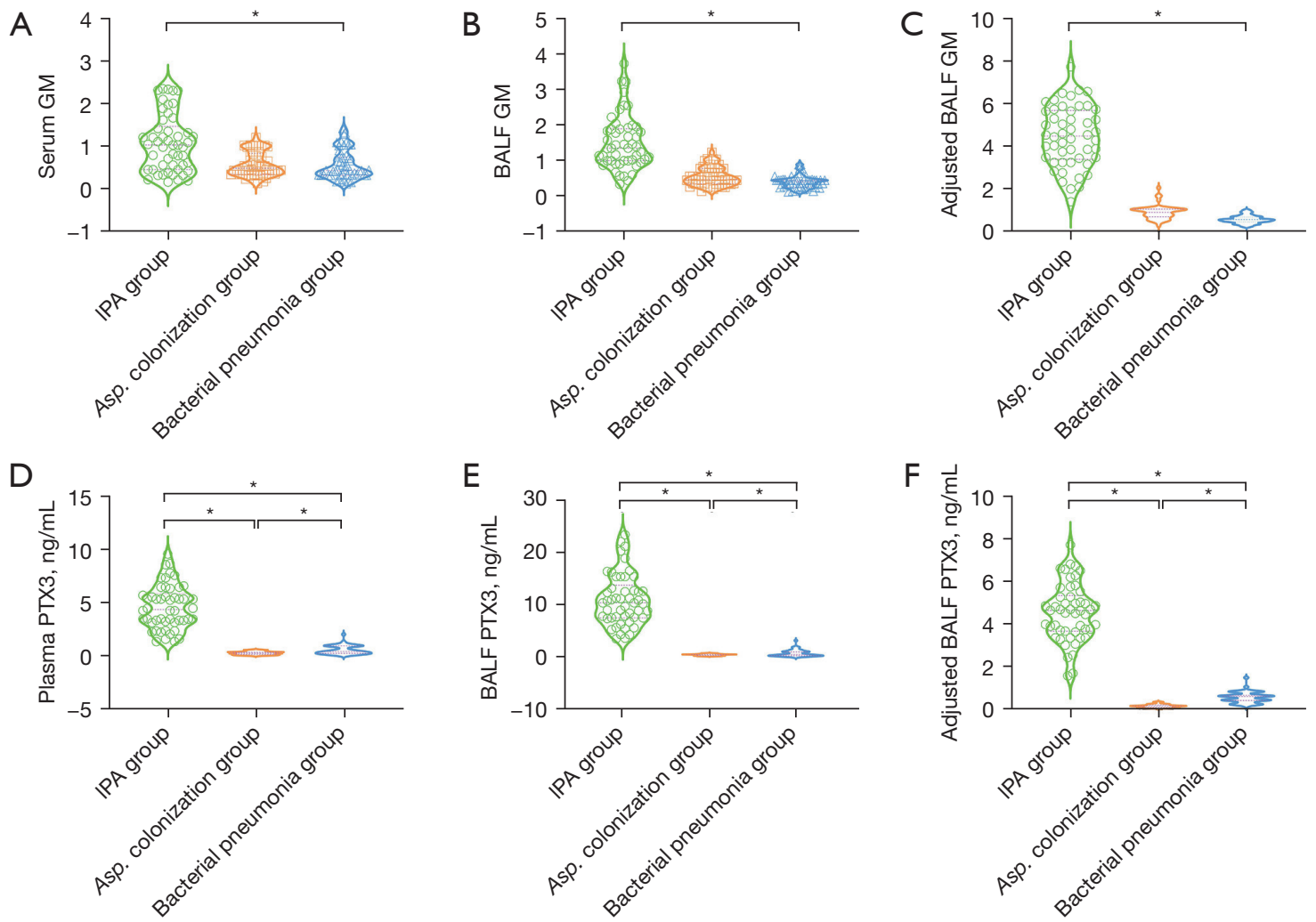

Figure 4 Comparation of GM and PTX3 among three different groups. (A) The concentration of serum GM in the three groups; (B) the concentration of BALF GM in the three groups; (C) the corrected concentration of BALF GM in the three groups; (D) the concentration of plasma PTX3 in the three groups; (E) the concentration of BALF PTX3 in the three groups; (F) the corrected concentration of BALF PTX3 in the three groups. *, $\mathrm{P}<0.05$. BALF, bronchoalveolar lavage fluid; GM, galactomannan; PTX3, pentraxin 3; IPA, invasive pulmonary aspergillosis.

which suggested that AA homozygosity could increase the risk of IPA [odds ratio (OR) 18.86; 95\% CI: 7.96-44.69; $\mathrm{P}<0.01]$. The AUC of SNP rs1840680 AA was 0.81, which corresponded to a sensitivity of $72.04 \%$ and a specificity of $88.18 \%$ (Figure S2). No significant difference in the genotypic frequencies of the other 2 SNPs was observed $(\mathrm{P}>0.05$; Table 4).

The PTX3 levels in plasma and BALF and the GM levels in serum and BALF were also detected according to different genotypes. There was no difference observed in the GM concentrations $(\mathrm{P}>0.05)$ between the AA homozygosity group and the $\mathrm{AG}+\mathrm{GG}$ genotype group $(\mathrm{P}>0.05)$, whereas both plasma PTX3 and BALF levels were lower in the AA homozygosity group than in the AG+GG genotype group $(\mathrm{P}<0.05$; Table 5$)$. This indicates that insufficient PTX3 is released in patients with the AA genotype, leading to decreased resistance to Aspergillus and increased susceptibility to IPA.

\section{Diagnostic efficiency of different algorithms for diagnosing IPA}

Based on the susceptible host, chest CT imaging, clinical symptoms, and 7 other factors (culture, microscopy, plasma PTX3 $>3.3 \mathrm{ng} / \mathrm{mL}$, adjusted BALF PTX3 $>14.5 \mathrm{ng} / \mathrm{mL}$, serum GM $>0.5$ ODI, and adjusted BALF GM $>2.5$ ODI), 6 algorithms for diagnosing IPA were established (Figure 7). It was demonstrated that PTX3 gene polymorphisms can meaningfully contribute to diagnosing IPA. An algorithm consisting of $P T X 3$ gene polymorphisms, adjusted BALF PTX3 $>14.5 \mathrm{ng} / \mathrm{mL}$, adjusted BALF GM $>2.5$ ODI, or a positive culture/microscopy result led to a sensitivity of $90.03 \%$ and a specificity of $97.09 \%$ (algorithm 6). Furthermore, the accuracy of algorithm 6 was potentially as high as $94.67 \%$, with an AUC of 0.94 , which was higher than that of the other 5 algorithms (Table 6, Table S2, and Figure S3). 

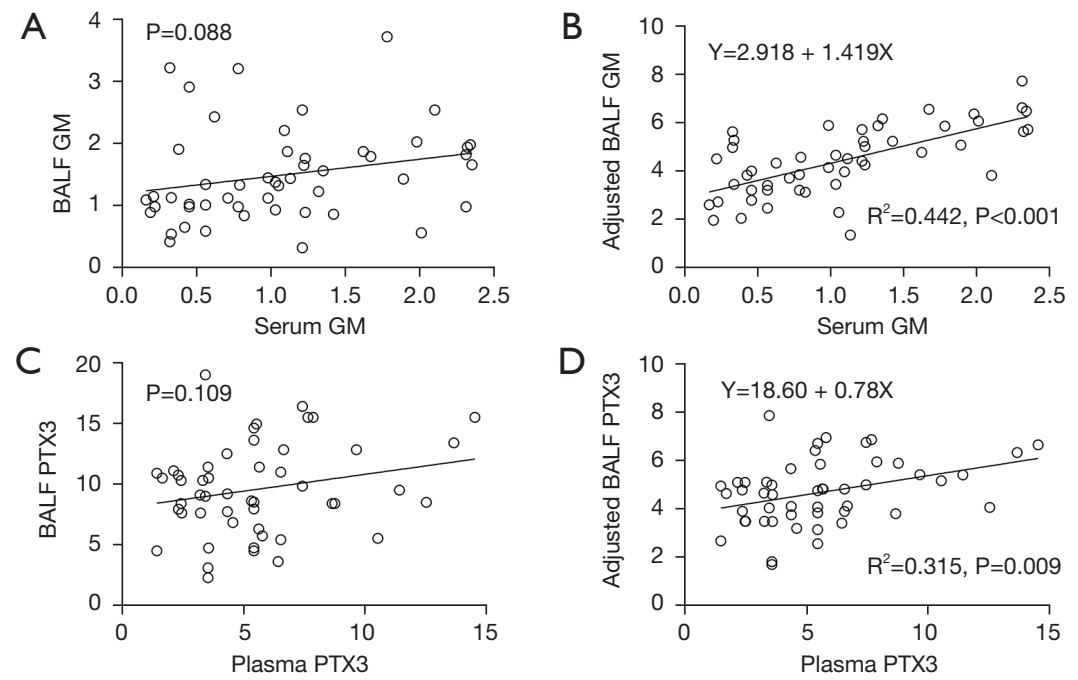

Figure 5 Correlations of GM and PTX3 in different samples among patients with invasive pulmonary aspergillosis (n=50). (A) Correlation of the serum GM and BALF GM; (B) correlation of the serum GM and adjusted BALF GM; (C) correlation of the plasma PTX3 and BALF PTX3; (D) correlation of the plasma PTX3 and adjusted BALF PTX3. BALF, bronchoalveolar lavage fluid; GM, galactomannan; PTX3, pentraxin 3; IPA, invasive pulmonary aspergillosis.

Table 2 Performance of galactomannan for diagnosing IPA at different cutoff values

\begin{tabular}{lcccccc}
\hline Variables & Sensitivity (\%) & Specificity (\%) & $95 \% \mathrm{Cl}$ & Youden index & Accuracy (\%) & AUC \\
\hline Serum GM $>0.5$ & 74.23 & 54.08 & $0.56-0.72$ & 0.28 & 60.67 & 0.64 \\
Serum GM $>1.0$ & 52.03 & 82.09 & $0.59-0.75$ & 0.34 & 72.03 & 0.67 \\
BALF GM $>0.5$ & 86.17 & 55.48 & $0.62-0.77$ & 0.42 & 64.77 & 0.71 \\
BALF GM $>0.8$ & 80.02 & 78.21 & $0.72-0.85$ & 0.58 & 78.67 & 77.06 \\
BALF GM $>1.0$ & 60.27 & 92.36 & $0.68-0.83$ & 0.53 & 0.79 \\
\hline
\end{tabular}

IPA, invasive pulmonary aspergillosis; AUC, area under the curve; BALF, bronchoalveolar lavage fluid; CI, confidence interval; GM, galactomannan.

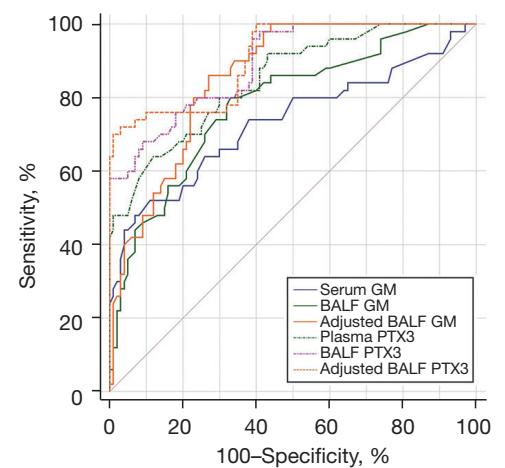

Figure 6 Receiver operating characteristic curves of the biomarkers for diagnosing invasive pulmonary aspergillosis. BALF, bronchoalveolar lavage fluid; GM, galactomannan; PTX3, pentraxin 3 .

\section{Discussion}

During the 5-year prospective study, PTX3 gene polymorphisms were detected, and the biomarkers related to IPA were tested from all of the 150 enrolled patients with autoimmune diseases. It was revealed that SNP rs1840680 and AA homozygosity could increase the risk for IPA. When the values of PTX3 and GM in BALF were adjusted by urea dilution, the rate of diagnostic accuracy for IPA was potentially increased. Six algorithms for diagnosing IPA were established, with the algorithm based on gene polymorphisms and the PTX3 value appearing to be the most reliable.

IPA is a refractory disease with a long period of treatment and a high mortality rate (1). Although a number of 
Table 3 Performance of different variables for diagnosing IPA

\begin{tabular}{|c|c|c|c|c|c|c|}
\hline Variables & Sensitivity (\%) & Specificity (\%) & $95 \% \mathrm{Cl}$ & Youden index & Cutoff & $A \cup C$ \\
\hline BALF GM & 80.08 & 67.03 & $0.71-0.85$ & 0.47 & 0.82 & 0.78 \\
\hline Adjusted BALF GM & 86.08 & 73.44 & $0.79-0.91$ & 0.59 & 2.51 & 0.85 \\
\hline Plasma PTX3 & 64.09 & 88.12 & $0.78-0.90$ & 0.52 & 3.34 & 0.85 \\
\hline Adjusted BALF PTX3 & 72.12 & 97.82 & $0.85-0.95$ & 0.69 & 14.51 & 0.91 \\
\hline
\end{tabular}

IPA, invasive pulmonary aspergillosis; AUC, area under the curve; BALF, bronchoalveolar lavage fluid; CI, confidence interval; GM, galactomannan; PTX3, pentraxin 3.

Table 4 Genotype distribution of single nucleotide polymorphisms in invasive pulmonary aspergillosis cases and controls

\begin{tabular}{|c|c|c|c|c|c|}
\hline SNP genotype & IPA $(n=50), n[\%]$ & $\begin{array}{l}\text { Aspergillosis colonization } \\
\qquad(\mathrm{n}=50), \mathrm{n}[\%]\end{array}$ & $\begin{array}{l}\text { Bacterial pneumonia } \\
\qquad(\mathrm{n}=50), \mathrm{n}[\%]\end{array}$ & OR $(95 \% \mathrm{Cl})$ & $P$ value \\
\hline \multicolumn{6}{|l|}{ rs1840680 } \\
\hline$A G$ & $6[12]$ & $21[42]$ & 19 [38] & $0.21(0.08-0.53)$ & $<0.01$ \\
\hline$G G+A G$ & $14[28]$ & $43[86]$ & $45[90]$ & $0.05(0.02-0.126)$ & $<0.01$ \\
\hline \multicolumn{6}{|l|}{ rs2305619 } \\
\hline AA & $28[56]$ & 26 [52] & $25[50]$ & $1.22(0.62-2.42)$ & 0.56 \\
\hline$G G+A G$ & $22[44]$ & 24 [48] & $25[50]$ & $0.82(0.41-1.62)$ & 0.56 \\
\hline \multicolumn{6}{|l|}{ rs3816527 } \\
\hline AA & $30[60]$ & $29[58]$ & $30[60]$ & $1.04(0.52-2.08)$ & 0.91 \\
\hline $\mathrm{AC}$ & 16 [32] & $18[36]$ & 17 [34] & $0.87(0.42-1.80)$ & 0.72 \\
\hline $\mathrm{CC}$ & $4[8]$ & $3[6]$ & 3 [6] & $1.36(0.37-5.07)$ & 0.65 \\
\hline$A C+A A$ & 46 [92] & 47 [94] & 47 [94] & 0.73 (0.19-2.73) & 0.66 \\
\hline $\mathrm{AC}+\mathrm{CC}$ & $20[40]$ & $21[42]$ & $20[40]$ & $0.96(0.48-1.92)$ & 0.91 \\
\hline
\end{tabular}

IPA, invasive pulmonary aspergillosis; OR, odds ratio; $\mathrm{Cl}$, confidence interval; SNP, single nucleotide polymorphisms.

diagnostic tools have been developed, the accurate diagnosis of IPA remains a challenge (15). Biomarkers in BALF, such as GM, have been recommended as promising indicators of the growth of Aspergillus, but the degree of BALF dilution is variable. Studies focused on drug concentrations in epithelial lining fluid (ELF) have demonstrated that urea dilution can be performed as an internal parameter to adjust the concentration of BALF $(16,17)$. Thus, the same approach was applied to adjust the values of GM and PTX3 in BALF; the adjusted BALF GM value was 2.5 ODI, which 
Table 5 The level of PTX3 and GM in different genotypes in IPA patients

\begin{tabular}{lccc}
\hline Variables & AA genotype $(n=36)$ & GG+AG genotype $(n=14)$ & P value \\
\hline PTX3 in plasma & $3.65(2.87-4.82)$ & $4.73(3.62-6.24)$ & 0.02 \\
PTX3 in BALF & $9.77(6.83-12.54)$ & $12.93(8.92-16.41)$ & $<0.01$ \\
GM in serum & $0.98(0.45-1.42)$ & $1.03(0.38-1.57)$ & 0.810 \\
GM in BALF & $1.41(0.97-1.94)$ & $1.36(1.03-1.87)$ & 0.740 \\
\hline
\end{tabular}

BALF, bronchoalveolar lavage fluid; GM, galactomannan; IPA, invasive pulmonary aspergillosis; PTX3, pentraxin 3.

Table 6 Performance of different algorithms for diagnosing IPA at different cutoff values

\begin{tabular}{lcccccc}
\hline Variables & Sensitivity (\%) & Specificity (\%) & $95 \%$ Cl & Youden index & Accuracy (\%) & AUC \\
\hline Algorithms 1 & 51.04 & 81.18 & $0.57-0.74$ & 0.32 & 74.04 & 0.66 \\
Algorithms 2 & 64.43 & 84.92 & $0.67-0.82$ & 0.49 & 78.71 & 0.75 \\
Algorithms 3 & 72.04 & 85.73 & $0.71-0.85$ & 0.58 & 0.79 \\
Algorithms 4 & 74.09 & 97.07 & $0.79-0.91$ & 0.71 & 89.33 & 0.86 \\
Algorithms 5 & 86.07 & 98.02 & $0.86-0.95$ & 0.84 & 0.83 .33 & 0.92 \\
Algorithms 6 & 90.03 & 97.09 & $0.88-0.97$ & 0.97 & 94.67 \\
\hline
\end{tabular}

IPA, invasive pulmonary aspergillosis; AUC, area under the curve; BALF, bronchoalveolar lavage fluid; Cl, confidence interval; GM, galactomannan.

accorded with our previous study (5).

PTX3 has been recognized as a soluble pattern recognition receptor (PRR) which can be detected while injury or inflammation is occurring (18). In addition, it also reveals that PTX3 has a nonredundant role in modulating the various effects or pathways involved in immune resistance to Aspergillus fumigatus, with its effects involving the activation of innate immune cells and the driving of protective adaptive immunity $(19,20)$. Therefore, PTX3 could be detected in both the plasma and BALF samples in the IPA group, and the levels were significantly higher than those in the control groups, which is consistent with the findings of Kabbani et al. (21).

Whether PTX3 gene polymorphisms are associated with the incidence of IPA is still controversial. The genotypic distributions from different hosts (with HSCT, solid organ transplantation, or chronic obstructive pulmonary disease) are not uniform $(7,14,22)$. Nevertheless, 3 SNPs (rs1840680, rs2305619, and rs3816527) in the PTX3 gene is worth studying. Our study indicated that the SNP rs1840680 AA homozygote was associated with a higher risk of IPA. However, the data on PTX3 SNPs and IPA are controversial $(6,7,14,22)$. It has been suggested that different genotypic distributions across disease settings may be one of the factors influencing the results of SNPs. In addition, racial/ethnic differences in metabolic phenotype can also be explained by differences in SNP distribution $(23,24)$. Thus, more parameters need to be combined to establish a reliable diagnostic algorithm.

The AspICU algorithm has been applied in clinical practice to identify patients with aspergillosis airway colonization, but no biomarker has been included because of low sensitivity. In this study, we applied the adjusted BALF PTX3 value, GM value, and PTX3 gene polymorphisms to modify the AspICU algorithm and established 6 algorithms for use in a diagnostic gradation criteria system. Thus, different algorithms may be selected by medical institutions to diagnose IPA, according to their specific conditions.

One new algorithm for diagnosing IPA based on PTX 3 gene polymorphisms and an adjusted BALF value was established in our study. However, limitations of the study exist. The autoimmune diseases present among the patients with IPA were SLE, ANCA-associated vasculitis, and dermatomyositis. Subgroup analysis could not be implemented due to the small sample size, and our research group is undertaking further research and analysis. The association between PTX3 genotype, the concentration of PTX3, and prognosis still needs further research. 


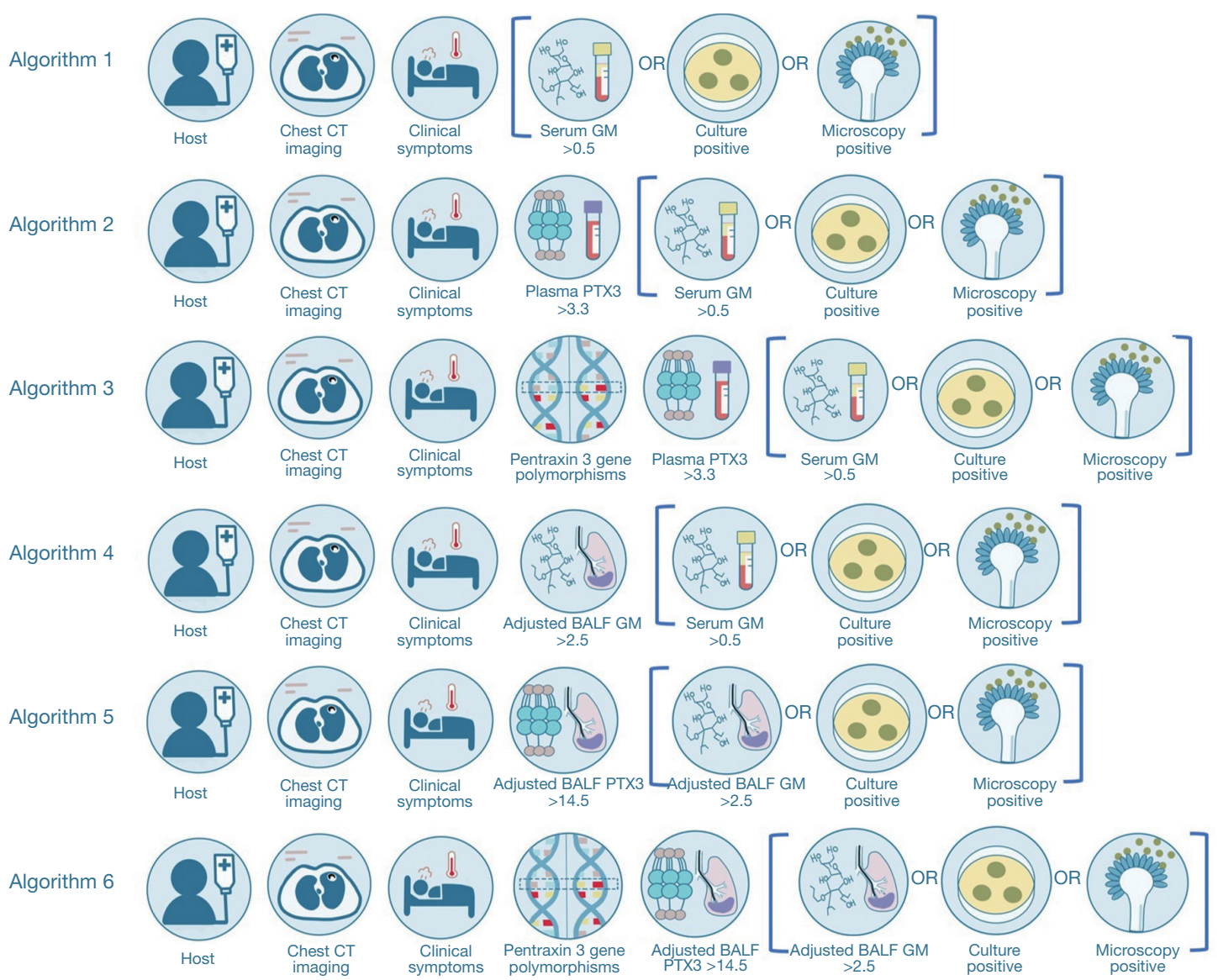

Figure 7 Algorithms for diagnosis of invasive pulmonary aspergillosis (drawn by Chunhui Xu). CT, computed tomography; GM, galactomannan; PTX3, pentraxin 3; BALF, bronchoalveolar lavage fluid.

\section{Conclusions}

It has been demonstrated that both PTX 3 gene polymorphisms and the adjusted value of PTX3 in BALF have value in the diagnosis of IPA. When applying an algorithm consisting of PTX 3 gene polymorphisms and PTX3 and GM-adjusted BALF values, the performance of diagnosing IPA is reliable.

\section{Acknowledgments}

On behalf of the research team, we would like to express our gratitude to all the members who participated in the data collection, samples testing and data's classification and analysis during the past 5 years.

Funding: This work was supported by the Scientific Research Project of Shanghai Municipal Health Bureau (No. 201840006) and the National Natural Science Foundation (grant number 81971990).

\section{Footnote}

Reporting Checklist: The authors have completed the STARD reporting checklist. Available at https://dx.doi. org/10.21037/atm-21-4017

Data Sharing Statement: Available at https://dx.doi. org/10.21037/atm-21-4017

Conflicts of Interest: All authors have completed the ICMJE uniform disclosure form (available at https://dx.doi. org/10.21037/atm-21-4017). The authors have no conflicts of interest to declare.

Ethical Statement: The authors are accountable for all aspects of the work in ensuring that questions related to the accuracy or integrity of any part of the work are appropriately investigated and resolved. The study was conducted in accordance with the declaration of Helsinki (as 
revised in 2013). The Ethics Committee of Shanghai Jiao Tong University approved the study (No. 2016-ClinicalRes-083), and written informed consent was obtained from all the inpatient participants or their next of kin.

Open Access Statement: This is an Open Access article distributed in accordance with the Creative Commons Attribution-NonCommercial-NoDerivs 4.0 International License (CC BY-NC-ND 4.0), which permits the noncommercial replication and distribution of the article with the strict proviso that no changes or edits are made and the original work is properly cited (including links to both the formal publication through the relevant DOI and the license). See: https://creativecommons.org/licenses/by-nc-nd/4.0/.

\section{References}

1. Patterson TF, Thompson GR 3rd, Denning DW, et al. Practice Guidelines for the Diagnosis and Management of Aspergillosis: 2016 Update by the Infectious Diseases Society of America. Clin Infect Dis 2016;63:e1-e60.

2. Ullmann AJ, Aguado JM, Arikan-Akdagli S, et al. Diagnosis and management of Aspergillus diseases: executive summary of the 2017 ESCMID-ECMM-ERS guideline. Clin Microbiol Infect 2018;24 Suppl 1:e1-e38.

3. Bassetti M, Azoulay E, Kullberg BJ, et al. EORTC/ MSGERC Definitions of Invasive Fungal Diseases: Summary of Activities of the Intensive Care Unit Working Group. Clin Infect Dis 2021;72:S121-7.

4. Blot SI, Taccone FS, Van den Abeele AM, et al. A clinical algorithm to diagnose invasive pulmonary aspergillosis in critically ill patients. Am J Respir Crit Care Med 2012;186:56-64.

5. Yu Y, Zhu C, Shen H, et al. Galactomannan detection in bronchoalveolar lavage fluid corrected by urea dilution for the diagnosis of invasive pulmonary aspergillosis among nonneutropenic patients. J Thorac Dis 2019;11:465-76.

6. Cunha C, Aversa F, Lacerda JF, et al. Genetic PTX3 deficiency and aspergillosis in stem-cell transplantation. $\mathrm{N}$ Engl J Med 2014;370:421-32.

7. Wójtowicz A, Lecompte TD, Bibert S, et al. PTX3 Polymorphisms and Invasive Mold Infections After Solid Organ Transplant. Clin Infect Dis 2015;61:619-22.

8. Kalil AC, Metersky ML, Klompas M, et al. Management of Adults With Hospital-acquired and Ventilator-associated Pneumonia: 2016 Clinical Practice Guidelines by the Infectious Diseases Society of America and the American Thoracic Society. Clin Infect Dis 2016;63:e61-e111.
9. Gordon C, Amissah-Arthur MB, Gayed M, et al. The British Society for Rheumatology guideline for the management of systemic lupus erythematosus in adults. Rheumatology (Oxford) 2018;57:e1-e45.

10. Yates M, Watts RA, Bajema IM, et al. EULAR/ERAEDTA recommendations for the management of ANCAassociated vasculitis. Ann Rheum Dis 2016;75:1583-94.

11. Aggarwal R, Rider LG, Ruperto N, et al. 2016 American College of Rheumatology/European League Against Rheumatism criteria for minimal, moderate, and major clinical response in adult dermatomyositis and polymyositis: An International Myositis Assessment and Clinical Studies Group/Paediatric Rheumatology International Trials Organisation Collaborative Initiative. Ann Rheum Dis 2017;76:792-801.

12. Du Rand IA, Blaikley J, Booton R, et al. British Thoracic Society guideline for diagnostic flexible bronchoscopy in adults: accredited by NICE. Thorax 2013;68 Suppl 1:i1-i44.

13. de Boer MG, Halkes CJ, van de Vosse E. PTX3 deficiency and aspergillosis. N Engl J Med 2014;370:1665-6.

14. He Q, Li H, Rui Y, et al. Pentraxin 3 Gene Polymorphisms and Pulmonary Aspergillosis in Chronic Obstructive Pulmonary Disease Patients. Clin Infect Dis 2018;66:261-7.

15. Bassetti M, Giacobbe DR, Grecchi C, et al. Performance of existing definitions and tests for the diagnosis of invasive aspergillosis in critically ill, adult patients: A systematic review with qualitative evidence synthesis. J Infect 2020;81:131-46.

16. Andersen CU, Sønderskov LD, Bendstrup E, et al. Voriconazole Concentrations in Plasma and Epithelial Lining Fluid after Inhalation and Oral Treatment. Basic Clin Pharmacol Toxicol 2017;121:430-4.

17. Wenzler E, Gotfried MH, Loutit JS, et al. MeropenemRPX7009 Concentrations in Plasma, Epithelial Lining Fluid, and Alveolar Macrophages of Healthy Adult Subjects. Antimicrob Agents Chemother 2015;59:7232-9.

18. Kang Y, Yu Y, Lu L. The Role of Pentraxin 3 in Aspergillosis: Reality and Prospects. Mycobiology 2020;48:1-8.

19. Lee YT, Gong M, Chau A, et al. Pentraxin-3 as a marker of sepsis severity and predictor of mortality outcomes: A systematic review and meta-analysis. J Infect 2018;76:1-10.

20. Moalli F, Doni A, Deban L, et al. Role of complement and $\mathrm{Fc}\{$ gamma $\}$ receptors in the protective activity of the long pentraxin PTX3 against Aspergillus fumigatus. Blood 2010;116:5170-80. 
21. Kabbani D, Bhaskaran A, Singer LG, et al. Pentraxin 3 levels in bronchoalveolar lavage fluid of lung transplant recipients with invasive aspergillosis. J Heart Lung Transplant 2017;36:973-9.

22. Fisher CE, Hohl TM, Fan W, et al. Validation of single nucleotide polymorphisms in invasive aspergillosis following hematopoietic cell transplantation. Blood 2017;129:2693-701.

Cite this article as: $\mathrm{Yu} \mathrm{Y,} \mathrm{Liu} \mathrm{C,} \mathrm{Zhu} \mathrm{C,} \mathrm{Zhong} \mathrm{H,} \mathrm{Gu} \mathrm{Z,}$ Xu C, Pan C, Xu Z, Wu Z, Wu W, Lu L. A novel algorithm for diagnosis of invasive pulmonary aspergillosis based on pentraxin 3 gene polymorphisms and its adjusted value among autoimmune diseases patients. Ann Transl Med 2022;10(1):17. doi: $10.21037 /$ atm-21-4017
23. Dedmon LE. The genetics of rheumatoid arthritis. Rheumatology (Oxford) 2020;59:2661-70.

24. McGraw J, Waller D. Cytochrome P450 variations in different ethnic populations. Expert Opin Drug Metab Toxicol 2012;8:371-82.

(English Language Editors: K. Gilbert and J. Gray) 

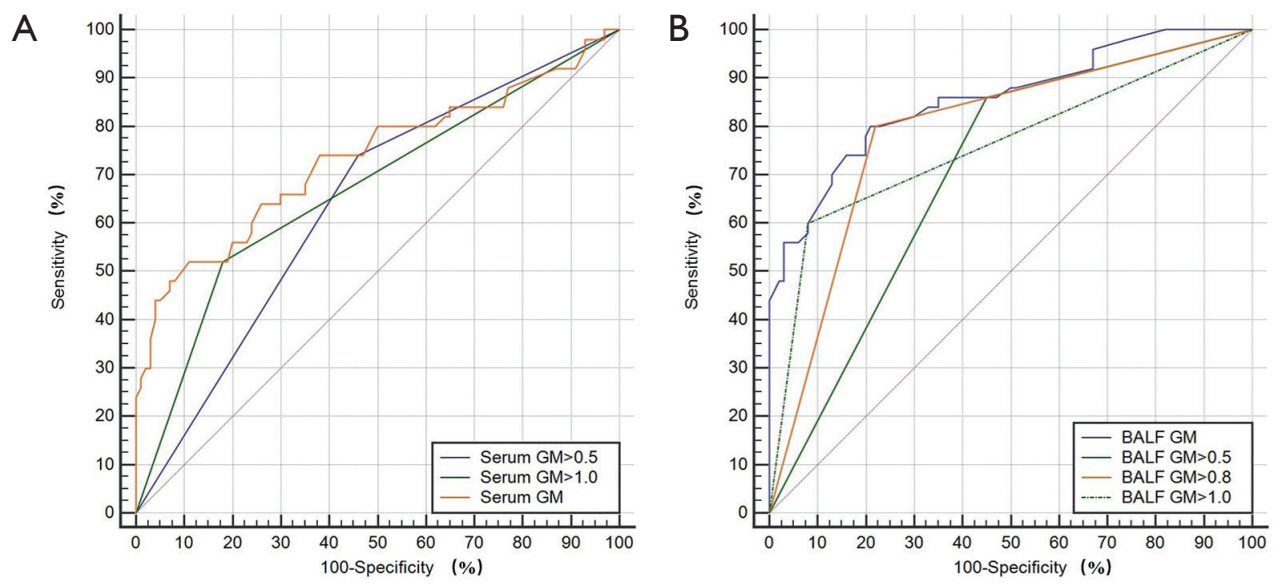

Figure S1 The receiver operating characteristic curves of galactomannan in diagnosing invasive pulmonary aspergillosis. (A) The receiver operating characteristic curve of serum galactomannan; (B) the receiver operating characteristic curve of bronchoalveolar lavage fluid galactomannan. BALF, bronchoalveolar lavage fluid; GM, galactomannan.

Table S1 Comparation of different variables in diagnosing IPA

\begin{tabular}{|c|c|c|c|c|}
\hline Variable & Difference between areas & SE & $95 \% \mathrm{Cl}$ & $P$ \\
\hline Serum_GM vs. adjusted BALF_GM & 0.12 & 0.05 & 0.03 to 0.22 & 0.02 \\
\hline Serum_GM vs. plasma_PTX3 & 0.12 & 0.06 & 0.01 to 0.23 & 0.04 \\
\hline Serum_GM vs. BALF_PTX3 & 0.16 & 0.06 & 0.04 to 0.27 & 0.01 \\
\hline BALF_GM vs. adjusted BALF_GM & 0.07 & 0.05 & -0.02 to 0.16 & 0.11 \\
\hline BALF_GM vs. plasma_PTX3 & 0.07 & 0.06 & -0.04 to 0.17 & 0.23 \\
\hline BALF_GM vs. BALF_PTX3 & 0.11 & 0.05 & 0.02 to 0.20 & 0.02 \\
\hline BALF_GM vs. adjusted_BALF_PTX3 & 0.12 & 0.05 & 0.03 to 0.22 & 0.01 \\
\hline Adjusted_BALF_GM vs. adjusted_BALF_PTX3 & 0.05 & 0.04 & -0.03 to 0.13 & 0.23 \\
\hline Plasma_PTX3 vs. BALF_PTX3 & 0.04 & 0.04 & -0.05 to 0.13 & 0.35 \\
\hline Plasma_PTX3 vs. adjusted_BALF_PTX3 & 0.06 & 0.04 & -0.02 to 0.14 & 0.17 \\
\hline BALF_PTX3 vs. adjusted_BALF_PTX3 & 0.02 & 0.04 & -0.06 to 0.09 & 0.66 \\
\hline
\end{tabular}

IPA, invasive pulmonary aspergillosis; BALF, bronchoalveolar lavage fluid; Cl, confidence interval; GM, galactomannan; PTX3, pentraxin 3; SE, standard error. 


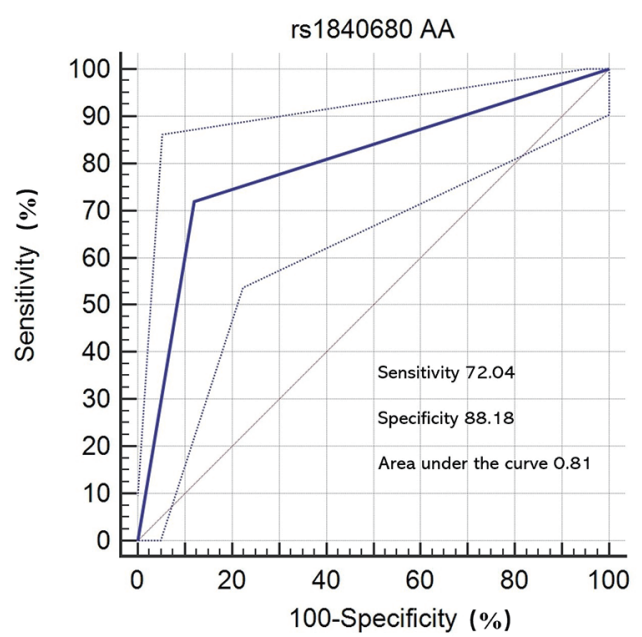

Figure S2 The receiver operating characteristic curves of rs1840680 AA in diagnosing invasive pulmonary aspergillosis.

Table S2 Comparation of different algorithms in diagnosing IPA

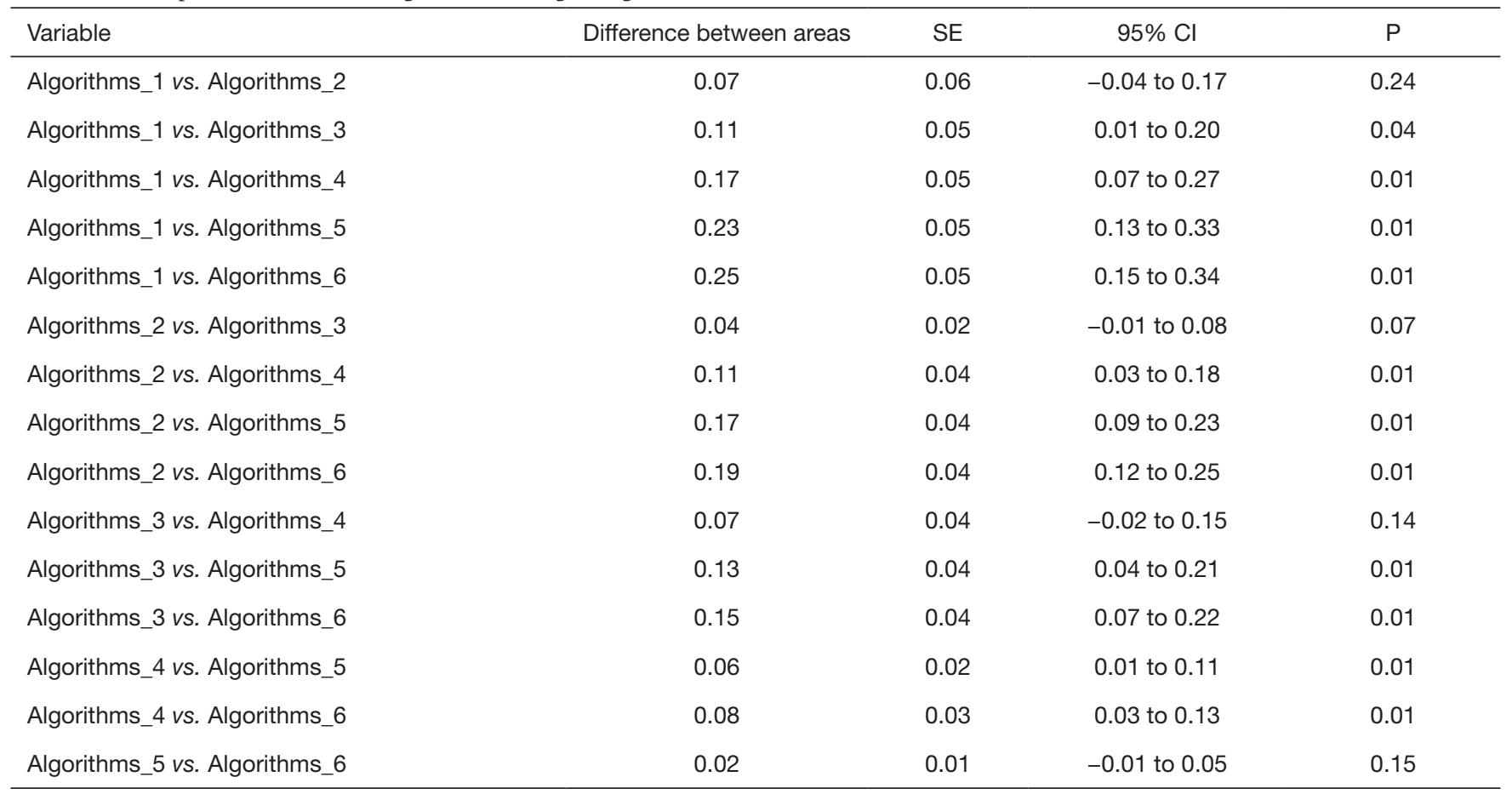

IPA, invasive pulmonary aspergillosis; $\mathrm{Cl}$, confidence interval; SE, standard error. 


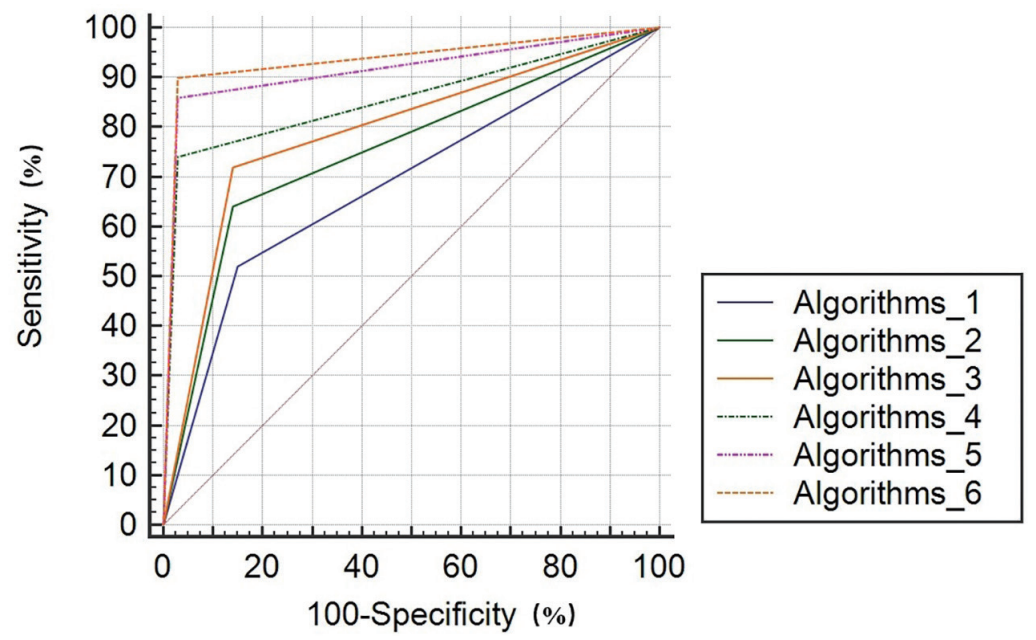

Figure S3 The receiver operating characteristic curves of different algorithms in diagnosing invasive pulmonary aspergillosis. 